\title{
Tara Kernel Fuzzy Clustering (TKFCM) for a Robust Adaptive Threshold Algorithm based on Level Set Method
}

\author{
Tara.Saikumar ${ }^{1}$, B.K.Anoop ${ }^{2}$, P.S.Murthy ${ }^{3}$ \\ ${ }^{1}$ Asst Professor, Dept of ECE, CMR Technical Campus, Hyderabad, India. \\ tara.sai437@gmail.com \\ ${ }^{2}$ Asst Professor, Dept of ECE, ACE, Shonaur, India. \\ anoopbk2000@gmail.com \\ ${ }^{3}$ Professor, Dept of CSE, CMRIT, Hyderabad India. \\ moorthypsmegmail.com
}

\begin{abstract}
To segment an image in thresholding method, a fixed threshold is not suitable if the background is rough here, we propose a new robust adaptive thresholding method using TKFCM. The method requires only one parameter to be selected and the adaptive threshold surface can be found automatically from the original image. A Robust adaptive thresholding scheme using adaptive tracking and morphological filtering. TKFCM algorithm computes the fuzzy membership values for each pixel. On the source of TKFCM the edge indicator function was redefined. Using the edge indicator function of an image was performed to extract the boundaries of objects on the origin of the pre-segmentation. Therefore, the proposed method is computationally efficient. The efficiency and accuracy of the algorithm is demonstrated. The above process of segmentation showed a considerable improvement in the evolution of the level set function. Our method is good for detecting large and small images concurrently. It is also efficient to denoise and enhance the responses of images with low local contrast can be detected.
\end{abstract}

\section{KEYWORDS}

Adaptive thresholding, Image segmentation, TKFCM, level set method

\section{INTRODUCTION}

Image segmentation is plays an important role in the field of image understanding, image analysis, pattern identification. The primary essential goal of the segmentation process is to partition an image into regions that are homogeneous (uniform) with respect to one or more self characteristics and features. Clustering has long been a popular approach to novel pattern recognition. Image segmentation is important in the field of image understanding, image analysis, and pattern recognition and computer vision. The principal goal of the segmentation process is to partition an image into regions that are homogeneous with respect to one or more characteristics and features. The fuzzy c-means (FCM)[1] algorithm, as a typical clustering algorithm, has been utilized in a wide range of engineering and scientific disciplines such as medicine imaging, bioinformatics, pattern recognition, and data mining. Given a data $X=\left\{x_{i} \ldots \ldots \ldots x_{n}\right\} \subset R^{p}$, the original FCM algorithm partitions $\mathrm{X}$ into $\mathrm{c}$ fuzzy subsets by minimizing the following objective function

$$
J_{m}(U, V) \equiv \sum_{i=1}^{c} \cdot \sum_{k-1}^{n} u_{i k}^{m}\left\|x_{i}-v_{i}\right\|^{2} \ldots \ldots \ldots \ldots \text { (1.1) }
$$

Where $\mathrm{c}$ is the number of cluster and selected as a specified Value in the paper, $\mathrm{n}$ the number of data points, $u_{k}$, the member of $x_{k}$ in class $i$, satisfying $\sum_{i-1}^{c} u_{i k}$, m the quantity controlling 
clustering fuzziness and $\mathrm{v}$ is set of control cluster centers or a prototypes $\left(v_{i} \in R^{p}\right)$. The function $J_{m}$ is minimized by the famous alternate iterative algorithm. Since the original FCM uses the squared-norm to measure inner product with an appropriate 'kernel' function, one similarity between prototypes and data points, it can only be effective in clustering 'spherical' clusters. And many algorithms are resulting from the FCM in order to cluster more general dataset. Traditional segmentation algorithms are effective on the extraction of rigid objects. But, due to the impact of lighting in imaging process, sometimes the boundaries of object we get are not real, especially on the process of objects with varied topology structure, such as image; the traditional algorithms can't determine the real boundaries.

The level set method [3-6] is based on geometric deformable model, which translates the problem of evolution 2-D (3-D) close curve (surface) into the evolution of level set function in the space with higher dimension to obtain the advantage in handling the topology changing of the shape. The level set method has had great success in computer graphics and vision. Also, it has been widely used in medical imaging for segmentation and shape recovery [7-8]. Firstly, as using the local marginal information of the image, it is difficult to obtain a perfect result when there's a fuzzy or discrete boundary in the region, and the leaking problem is inescapably appeared; Secondly, solving the partial differential equation of the level set function requires numerical processing at each point of the image domain which is a time consuming process; Finally, if the initial evolution contour is given at will, the iteration time would increase greatly, too large or too small contour will cause the convergence of evolution curve to the contour of object incorrectly.

In the paper, based on the new deviational level set method, the edge indicator function was weighted to improve the ability of detecting fuzzy boundaries of the object. At the same time, the FCM algorithm [9-10] was applied to obtain the appropriate initial contour of evolution curve, so as to get the accurate contour of object and reduce the evolution time.

\section{ADAPTIVE THRESHOLD ALGORITHM}

We adapt this technique with some optimization. The following outlines Hoover's adaptive thresholding:

1) Binarize the image with a single threshold $T$

2) Thin the thresholded image

3) Erase all branchpoints in the thinned image

4) All remaining endpoints are placed in the probe

queue and are used as a starting point for tracking

5) Track the region with threshold $T$

6) If the region passed testing, $T=T$-1, go to 5)

The testing in step 6) is some constraints to guarantee the region is image segment .Very small segments that remain after thresholding will be "size-filtered". But this step is preceded by the application of morphological filter to fill the small gaps between images.

\section{Tara Kernel Fuzzy C- Means Clustering}

Define a nonlinear map as $\phi_{\bullet}: x \rightarrow \phi(x) \in F$, where $x \in X . X$ denotes the data space and $F$ is the transformed feature space with higher even infinite dimensions. TKFCM minimized the following objective function: $J_{m}(U, V) \equiv \sum_{i-1}^{c} \cdot \sum_{k-1}^{n} u_{i k}^{m}\left\|\phi\left(x_{i}\right)-\phi\left(v_{i}\right)\right\|^{2}$ 
Where

$\left\|\phi\left(x_{i}\right)-\phi\left(v_{i}\right)\right\|^{2}=K\left(x_{k}, x_{k}\right)+K\left(v_{i}, v_{i}\right)-2 K\left(x_{k}, v_{i}\right)$

Where $K(x, y)=\phi(x)^{T} \phi(y)$ is an inner product of the kernel function If we adopt the Gaussian function as a kernel function, $K(x, y)=\exp \left(-\|x-y\|^{2} / 2 \sigma^{2}\right)$, then $K(x, x)=1$. according to Eq. (2.3), Eq. (2.2) can be rewritten as

$$
J_{m}(U, V) \equiv 2 \sum_{i=1}^{c} \cdot \sum_{k=1}^{n} u_{i k}^{m}\left(1-k\left(x_{k}, v_{i}\right)\right) \cdot \cdots \cdots \cdots
$$

Minimizing Eq. (2.4) under the constraint of, $u_{i k}, m>1$. We have

$$
\begin{aligned}
& u_{i k}=\frac{\left(1 /\left(1-K\left(x_{k}, v_{i}\right)\right)\right)^{1 /(m-1)}}{\sum_{j=1}^{c}\left(1 /\left(1-K\left(x_{k}, v_{i}\right)\right)\right)^{1 /(m-1)}} \\
& v_{i}=\left[\frac{\sum_{k=1}^{n} u_{i k} K\left(x_{k}, v_{i}\right) x_{k}}{\sum_{k=1}^{n} u_{i k}^{m} K\left(x_{k}, v_{i}\right)}\right]^{1 / 2} \ldots \ldots . . .
\end{aligned}
$$

Here we now utilize the Gaussian kernel function for Straightforwardness. If we use additional kernel functions, there will be corresponding modifications in Eq. (2.5) and (2.6).

In fact, Eq.(2.3) can be analyzed as kernel-induced new metric in the data space, which is defined as the following

$$
d(x, y) \underline{\underline{\Delta}}\|\phi(x)-\phi(y)\|=\sqrt{2(1-K(x, y))}
$$

And it can be proven that $d(x, y)$ is defined in Eq. (2.7) is a metric in the original space in case that $K(x, y)$ takes as the Gaussian kernel function. According to Eq. (6), the data point $x_{k}$ is capable with an additional weight $K\left(x_{k}, v_{i}\right)$, which measures the similarity between $x_{k}$ and $v_{i}$ and when $x_{k}$ is an outlier i.e., $x_{k}$ is far from the other data points, then $K\left(x_{k}, v_{i}\right)$ will be very small, so the weighted sum of data points shall be more strong.

The full explanation of TKFCM algorithm is as follows:

\section{IKFCM Algorithm:}

Step 1: Select initial class prototype $\left\{v_{i}\right\}_{i=1}^{c}$.

Step 2: Update all memberships $u_{i k}$ with Eq. (2.5).

Step 3: Obtain the prototype of clusters in the forms of weighted average with Eq. (2.6).

Step 4: Repeat step 2-3 till termination. The termination criterion is $\left\|V_{\text {new }}-V_{\text {old }}\right\| \leq \varepsilon$.

Where $\|\cdot\|$ is the Euclidean norm. $V$ is the vector of cluster centers $\varepsilon$ is a small number that can be set by user (here $\varepsilon=0.01$ ). 


\section{The LeVel SeT MeTHOD}

The level set method was invented by Osher and Sethian [3] to hold the topology changes of curves. A simple representation is that when a surface intersects with the zero plane to give the curve when this surface changes, and the curve changes according with the surface changes. The heart of the level set method is the implicit representation of the interface. To get an equation describing varying of the curve or the front with time, we started with the zero level set function at the front as follows:

$$
\phi(x, y, t)=0, \text { if }(x, y) \in 1
$$

Then computed its derivative which is also equal to zero

$$
\frac{\partial \phi}{\partial t}+\frac{\partial \phi}{\partial x} \cdot \frac{\partial x}{\partial t}+\frac{\partial \phi}{\partial y} \cdot \frac{\partial y}{\partial t}=0
$$

Converting the terms to the dot product form of the gradient vector and the $x$ and $y$ derivatives vector, we go

$$
\frac{\partial \phi}{\partial t}+\left(\frac{\partial \phi}{\partial x} \cdot \frac{\partial x}{\partial t}\right) \cdot\left(\frac{\partial \phi}{\partial y} \cdot \frac{\partial y}{\partial t}\right)=0 \ldots \ldots \ldots
$$

Multiplying and dividing by $\nabla \phi$ and taking the other part to be $F$ the equation was gotten as follows:

$$
\frac{\partial \phi}{\partial t}+F|\nabla \phi|=0 \ldots \ldots \ldots \ldots
$$

According to literature [9]11], an energy function was defined:

$$
E(\phi)=\mu E_{\text {int }}(\phi)+E_{\text {ext }}(\phi)
$$

Where $E_{\text {ext }}(\phi)$ was called the external energy, and $E_{\text {int }}(\phi)$ was called the internal energy. These energy functions were represented as:

$$
\begin{aligned}
E_{\text {int }}(\phi) & =\int_{\Omega} \frac{1}{2}(\nabla \phi-1)^{2} d x d y . \\
E_{\text {ext }}(\phi) & =\lambda L_{g}(\phi)+v A_{g}(\phi) . \\
L_{g} & =\int_{\Omega} g \delta(\phi)|\nabla \phi| d x d y \ldots . \\
A_{g} & =\int_{\Omega} g H(-\phi) d x d y \ldots \ldots \\
g & =\frac{1}{1+\left|\nabla G_{\sigma} * I\right|} \ldots \ldots \ldots . .
\end{aligned}
$$

Where $L_{g}(\phi)$ was the length of zero level curve of $\phi$; and $A_{g}$ could be viewed as the weighted area; I was the image and $g$ was the edge indicator function. In conventional(traditional) level set methods, it is numerically necessary to keep the evolving level set function close to a signed distance function[14][15]. Re-initialization, a technique for occasionally re-initializing the level set function to a signed distance function during the evolution, has been extensively used as a numerical remedy for maintaining stable curve evolution and ensuring desirable results.

From the practical viewpoints, the re-initialization process can be quite convoluted, expensive, and has subtle side effects [16]. In order to overcome the problem, Li et al [8] proposed a new variational level set formulation, which could be easily implemented by simple finite difference scheme, without the need of re-initialization. The details of the algorithm are in the literature [8]. However, because only the gradient information was imposed in the edge indicator function, Li's method has a little effect on the presence of fuzzy boundaries. 
In the paper, a innovative method was proposed to modify the algorithm. The original image was partitioned into some sub images by TKFCM. The fuzzy boundary of each sub image was weighted by $\alpha$, the edge indicator function was redefined:

$g^{\prime}=g+\alpha \cdot g_{2} \ldots \ldots \ldots \ldots \ldots . . . .(4.11)$

Where $g_{2}=\frac{1}{1+\left|\nabla G_{\sigma} * I_{1}\right|}$

$I_{1}$ Was the image after clustering. The iterative equation

of level set functional was:

$\left.\frac{\left(\phi^{n+1}-\phi^{n}\right)}{\tau}=\mu\left[\Delta \phi-d i \psi\left(\frac{\nabla \phi}{\nabla \phi}\right)\right]+\lambda \not \phi\right) \operatorname{di}\left(g^{\prime} \frac{\nabla \phi}{\nabla \phi}\right)+v g^{\prime} \delta(\phi)$

Taking $g^{\prime}=g+\alpha \cdot g_{2}$ into 4.12

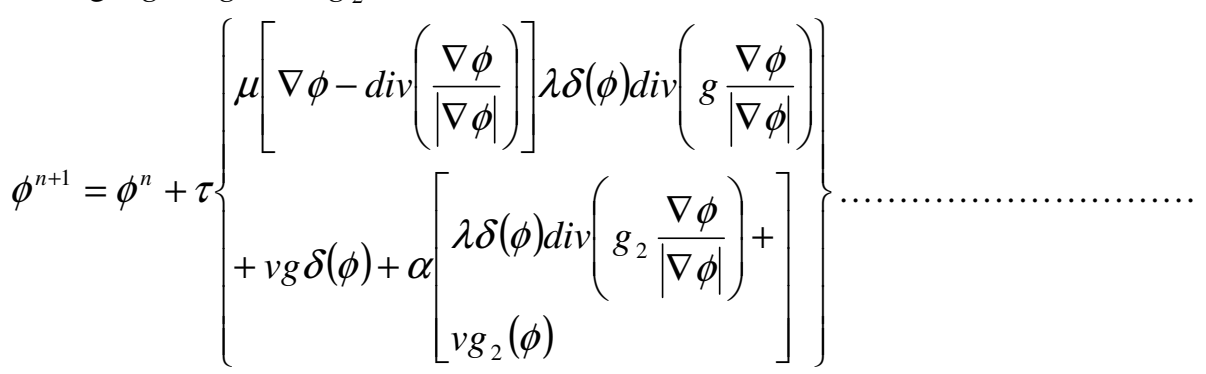

Where $\alpha \in[0,1]$. When processing images of weak boundary or low contrasts, a bigger $\alpha$ was taken; otherwise, a smaller $\alpha$ was taken.

\section{The Generation Of Initial Contour Curve}

On the basis of TKFCM clustering in image segmentation, the over segmentation usually exists. In this paper, the result of TKFCM was used as initial contour curve, and the automated initialization of deformation model was finished. For all the pixels in each cluster i.e. white matter, if 4 neighbourhoods included the heterogeneous pixel, the pixel was regarded as candidate boundary point. So the algorithm of curve tracing [17] was proposed. The exterior boundary of the cluster was tracked in the candidate boundary points. Finally, the closed curve was obtained. The candidate boundary points, whose Euclidean distances to the origin coordinates were shortest, were chosen as initiation points of curve tracing. The steps of image segmentation with adapted level set method were as follows:

Step1. Set the number of clusters, then the original image was processed with TKFCM, and calculate the $g_{2}$.

Step2. Choose one cluster, evaluate the inside area with $-\rho$ and the outside area with $+\rho, \rho$ is a plus constant. The boundary of the area is set to 0 . The region of interest is defined initial contour.

Step3. Minimize the overall energy functional with 4.13 formula.

\section{EXPERIMENTAL RESULT}

The segmentation of image takes an important branch in the surgery navigation and tumor radiotherapy. However, due to medical imaging characteristics, the low contrast and fuzzy boundary is usually occurred in the images. In the experiment, the samples of images are taken from internet as shown in Figure i. 


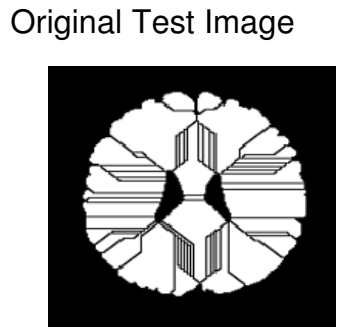

Original Test Imaqe

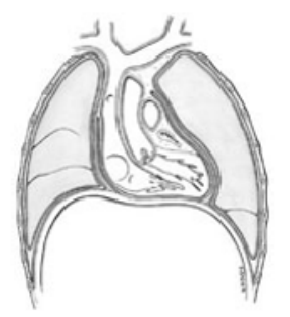

Original Test Image

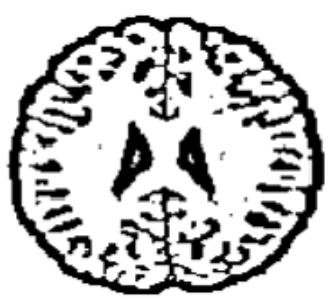

igure $\mathrm{i}$

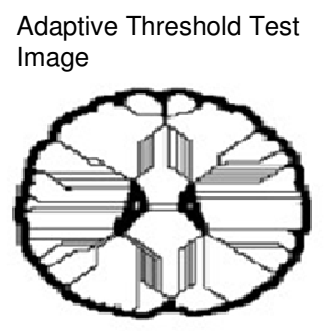

Adaptive Threshold Test Image

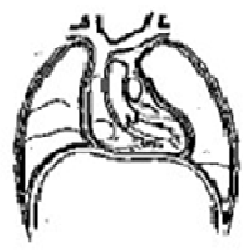

Adaptive Threshold Test Image

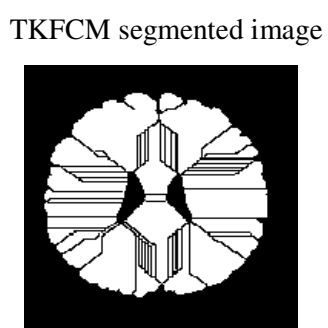

TKFCM segmented image

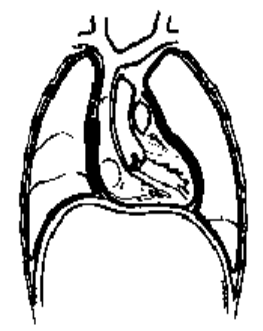

TKFCM segmented
Proposed Final contour, 300iteration

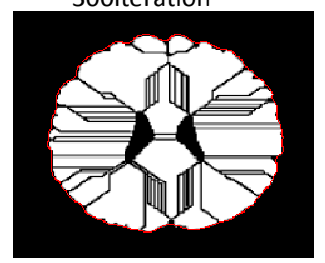

Proposed Final contour, 300iteration

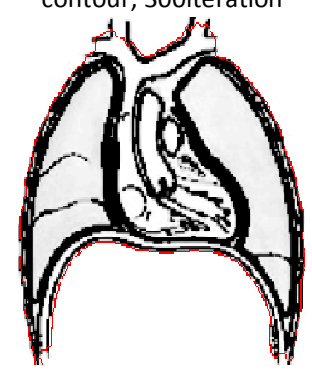

Proposed Final contour, 300iteration

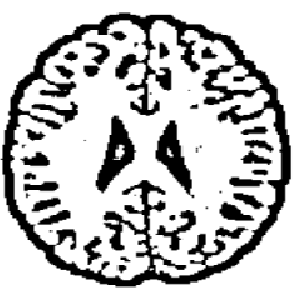

Figure ii

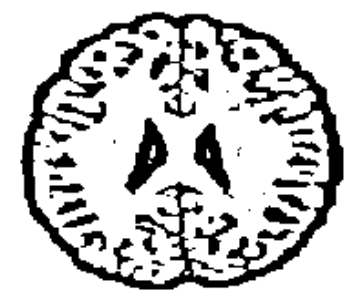

Figure iii

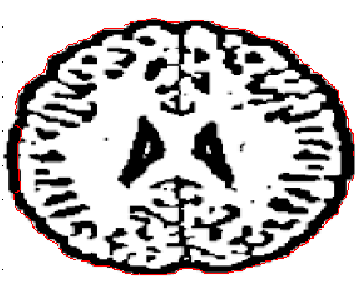

Figure iv

Figure $i$ are the original test images,

Figure ii are the results of Adpative threshold image.

Figure iii are the results of TKFCM clustering, to extracting the white matter.

Figure iv are the results of final contour with proposed method.

With the enhanced the approximate contour of white matter was got by TKFCM algorithm shown in Figure ii. The snooping of regions else appear as a result of the in excess of segmentation.

The initial evolution curve was obtained by the automated initialization. Because of the improved edge indicator function, the curve regularly evolved to the object boundaries in the process of evolution. The result established that the improved algorithm can extract the contour of object enhanced at same time, because the curve has been converged to the narrow region the object boundaries extraction could not be implemented with Li's method. But the enhanced method solved this problem better. On the similar computing proposal, under a $3.0 \mathrm{GHz}$ Pentium iv PC with 1 GB RAM on board, the average processing time of improved method was 9.6s, and that was $30.3 \mathrm{~s}$ with Li's method. The evolution time was greatly reduced.

The plot shows about the time iteration of level set method is evolve is as shown in figure $\mathrm{v}$ below. It is the comparison between FCM and Tara KFCM in terms of cpu time. 


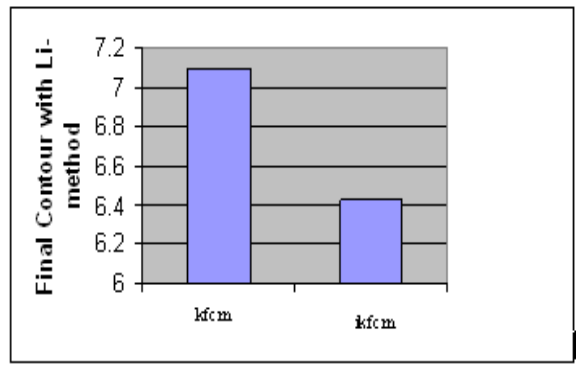

Figure v KFCM and TKFCM in terms of cpu time.

\section{DISCUSSIONS}

The need of the re-initialization is completely eliminated by the proposal of Chunming $\mathrm{Li}$, for pure partial differential equation driven level set methods, the variational level set methods. It can be easily implemented by using simple finite difference method and is computationally more efficient than the traditional level set methods. But, in this algorithm, the edge indicator has little effect on the low contrast image. So it is hard to obtain a perfect result when the region has a fuzzy or discrete boundary. Meanwhile, the initial contour of evolution needs to be determined by manual, and it has the shortcomings of time consuming and user intervention.

In this paper, we projected a new method to transform the algorithm. The original image was partitioned with adaptive algorithm, TKFCM, and the controlled action of the edge indicator function was increased. The result of TKFCM segmentation was used to obtain the initial contour of level set method. With the new edge indicator function, results of image segmentation showed that the improved algorithm can exactly extract the corresponding region of interest. Under the same computing proposal, the average time cost was lower. Alternatively the TKFCM clustering is sensitive to noise; some redundant boundaries were appeared in the candidates. Consecutively to solve this problem, the algorithm of curve tracing was proposed.

\section{CONCLUSIONS}

In conclusion, the results of this study confirmed that the combination of adaptive algorithm, TKCM with the level set methods could be used for the segmentation of low contrast images. The method has the advantages of no reinitialization, automation, and reducing the number of iterations. The validity of new algorithm was verified in the process of exacting different images. In the future research, the effect of priori information on the object boundary extraction with level set method, such as boundary, shape, and size, would be further analyzed. At the same time, the performance of image segmentation algorithms would be improved by reconstruction of classic velocity of level set method.

\section{REFERENCES}

[1] J.C.Bezdek, Pattern Recognition with Fuzzy Objective Function Algorthims,Plenum Press ,New York, 1981.

[2] K.L. Wu,M.S. Yang,Alternative c-means clustering algorthims,Pattern Recognition voI.35,pp.22672278,2002 .

[3] L.Zhang,W.D.zhou,L.C.Jiao.Kernel clustering algorthim, Chinese J. Computers, voI25(6),pp.587 590,2002(in chinese).

[4] Osher S, Sethian J.A,Fronts propagating with curvature dependent speed: algorthim's based on the Hamilton-Jacobi formulation.Journal of Computational Physics, 1988,pp. 12-49.

[5] Malladi,R,Sethain,J., and Vemuri,B., Shape modelling with front propagation: A level set approach.IEEE Trans.Pattern Anal.Mach.Intell, 1995, pp.158-175. 
[6] Staib,L., Zeng,X., Schultz,R., and Duncan,J., Shape constraints in deformable models. Handbook of Medical

Imaging,Bankman,1.,ed.,2000,pp.147-157

[7] Leventon,M., Faugeraus,O., Grimson,W., and Wells,W.,Level set based segmentation with intensity and curvature priors. Workshop on Mathematical Methods in Biomedical Image Analysis Proceedings,2000,pp.4-11.

[8] Paragios , Deriche R,Geodesic active contours and level sets for the detection and tracking of moving objects. IEEE Transaction on pattern Analsis and Machine In telligence,2000,pp.266-280.

[9] Vese L A,Chan T F, A multiphase level set frame wor for image segmentation using the mumford and shah model. International Journal of Computer Vision,2002,pp.271-293.

[10] Shi Yanggang,Karl W C, Real-time tracking usin g level set, IEEE Computer Society Conference on Computer Vision and Pattern Recognition,2005,pp,34-42

[11] Li Chunming, xu Chengyang,Gui Changfeng,et al, Level set evolution without re-initialization:a new varitional formulation.IEEE Computer Society Conference on Computer Vision and pattern Recognition,2005,pp.430-436.

[12] Sethain, 1, Level set Methods and Fast Marching Methods.Cambridge University Press 1999.

[13] Dunn, 1C., A fuzzy relative of the ISODATA process and its use in detecting compact well-separated Clusters.J.Cybern., 1973,pp.32-57.

[14] Bezedek,J., A convergence thheorem for the fuzzy ISODATA clustering algorthims. IEEE Trans.Pattern Anal.Mach.Intell., 1980,pp 78-82.

[15] S.Osher and R.Fedkiw, level set methods and Dynamic implicit surfaces,Sp[ringer,2002,pp.112-113.

[16] D.Peng,B?Merrimam,S.Osher,H.zhao, and M.Kang, A PDE- based fast local level set method, J.Comp.Phys,1996,pp.410-438.

[17] J.Gomes and O.Faugeras, Reconciling distance functions and Level Sets J.Visiual Communic. And Imag. Representation, 2000, pp.209-223.

[18] Mcinerney T, Terzopouls D,Deformable models in medical image analysis: a survey. Medical Analysis,1996,pp.91-108.

[19] Dao-Qiang Zhang, Song-CanChen,Clustering in completed data usig Kernel-based fuzzy c-means algorthim. Neural Processing Letters Volume 18,Issue 3(december 2003) Pages: 155-162, Year of Publication:2003.

[20] F.H.Y. Chan, F.K. Lam, Hui Zhu, "Adaptive Thresholding By Variational Method”, IEEE Transactions on Image Processing, vol.7, no. 3, pp. 468-473, March 1998.

\section{Authors}

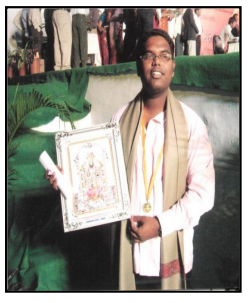

Tara.Saikumar was born in 1987 in Hyderabad, India. He received the B.Tech. degree in Electronics and Communication Engineering from Joginpally .B.R. Engineering College, Jawaharlal Nehru Technical University, Hyderabad in 2008, and the M.Tech. degree from Kakatiya Institute of Technology and Science (KITS), Warangal, India, in 2010 . He is the university topper in his M.Tech, KITS, Warangal and also received gold medal from Dr.D.N.Reddy vice-chancellor JNTUH in his B.Tech for his excellence2004-2008. He has more than 35 papers in his credit, International journal, International and National Conferences. He is a life member of IACSIT, and UAEEE. He got Best paper award in 2011.

$\mathrm{He}$ is currently a Asst Professor with the Dept of Electronics and Communication Engineering, CMR Technical Campus, Hyderabad. His research interests include digital image processing, digital signal processing, communication and neural network.

P.Sreenivasa Murthy obtained B.E., (CSE) from Osmania University, Hyderabad in 1995, M.E., (CSE) Sathyabama University, Chennai in 2006 and presently pursing Ph.D from Sant Gadge Baba Amaravati University, Amaravati. Presently working as Professor in the Dept. of Computer Science, CMR Institute of Technology, Hyderabad since Jul'2010. Life Member of ISTE, Member of International Association of Computer Science and Information Technology (IACSIT). Published a text book on Data Structures through C++. Expert Committee member for CDAC, Hyderabad. 